Itinéraires Itinéraires

Littérature, textes, cultures

2013-1 | 2013

La fiction aujourd'hui

\title{
D'un retour de flamme pour la fiction romanesque
}

La narration buissonnière selon Jean Echenoz

\section{Frank Wagner}

\section{(2) OpenEdition}

Journals

Édition électronique

URL : http://journals.openedition.org/itineraires/783

DOI : 10.4000/itineraires.783

ISSN : 2427-920X

Éditeur

Pléiade

\section{Édition imprimée}

Date de publication : 1 octobre 2013

Pagination : 29-49

ISBN : 978-2-343-01791-4

ISSN : $2100-1340$

Référence électronique

Frank Wagner, «D'un retour de flamme pour la fiction romanesque », Itinéraires [En ligne],

2013-1 | 2013, mis en ligne le 01 octobre 2013, consulté le 19 avril 2019. URL : http://

journals.openedition.org/itineraires/783; DOI : 10.4000/itineraires.783

\section{(c) (i) (9)}

Itinéraires est mis à disposition selon les termes de la licence Creative Commons Attribution - Pas d'Utilisation Commerciale - Pas de Modification 4.0 International. 


\section{D'un retour de flamme pour la fiction romanesque}

La narration buissonnière selon Jean Echenoz ${ }^{1}$

\section{Résumé}

À partir de l'étude d'un exemple concret, le début du chapitre 28 de Je m'en vais, cet article vise à clarifier les tenants et les aboutissants de la renarrativisation à l'œuvre dans nombre de fictions romanesques contemporaines. L'analyse successive de l'architexte, de l'intertexte et du métatextuel permet de mieux cerner un intense travail de modélisation esthétique, avant d'aboutir à l'examen de ses prolongements ethnologiques et ontologiques. Est ainsi mis en lumière un exemple de formalisme spéculatif, représentatif des tours et des détours du narratif qui caractérisent le roman d'aujourd'hui.

Mots clés : fiction contemporaine, renarrativisation, modélisation esthétique, transtextualité, formalisme spéculatif

\section{Abstract}

Taking as starting-point a concrete example, the beginning of chapter 28 of I'm off, this article seeks to clarify the ins and outs of the renarrativisation deployed in many contemporary novelistic fictions. It is possible, by successive analyses of the architext, the intertext and the metatextual to circumscribe more precisely this intense effort in the field of aesthetic modeling. This analysis sheds light on a case of speculative formalism which is representative of the tricks and detours of narrative and fiction which are characteristic of the novel today.

Keywords: contemporary fiction, renarrativisation, aesthetic modeling, transtextuality, speculative formalism

1. Lecture d'une séquence de Je m'en vais (Jean Echenoz, Je m 'en vais, Paris, Minuit, 1999 [Prix Goncourt], puis 2001, coll. « Minuit Double »). Toutes les indications de pagination renverront à la réédition en collection de poche. 


\section{Les « dessous » d'une œuvre}

Depuis une décisive étude d'Aaron Kibedi Varga ${ }^{2}$, l'idée d'une renarrativisation du roman contemporain a eu progressivement tendance, au fil de ces dernières décennies, à se figer en une forme de lieu commun critique, largement cautionné par les écrivains eux-mêmes. On peut y lire le symptôme d'un « retour d'affection » pour le roman, dont témoigne par exemple le constat rétrospectif formulé par Jean Echenoz, au cours d'un entretien paru en $2004^{3}$ :

Au début des années quatre-vingt, et je faisais partie de cette mouvance, il y a eu un retour d'affection pour le roman qui était un peu éclipsé pendant les années soixante-dix. De façons très diverses, c'étaient des déclarations d'amour au roman. Elles avaient des racines qui allaient souvent chercher dans les formes dites mineures, que ce soit le fantastique, le policier, la science-fiction, etc.

Le flirt souvent poussé de nombreux écrivains de la fin du $\mathrm{Xx}^{\mathrm{e}}$ siècle - Jean Echenoz, certes, mais aussi Antoine Volodine, Marie NDiaye, Tanguy Viel, Éric Laurrent, etc. - avec la littérature « de genre(s) », dans le but de renouer avec le plaisir de raconter des histoires, après une voire deux décennies d'ascèse scripturaliste, est en effet notoire. Envisagé au plus haut niveau de généralité, ce retour au/du récit, valorisant la fiction au sens fort du terme, pourrait à première vue paraître témoigner d'une attitude à quelque degré régressive, comme s'il s'était agi, à peu de chose près, de reprendre le roman là où l'avaient trouvé les Nouveaux Romanciers aux débuts de leurs expérimentations sur le genre. Hypothèse inconfortable, voire inquiétante, que semble indirectement cautionner la suite de la déclaration de Jean Echenoz :

Ce mouvement-là me paraît avoir éclaté. Le rapport au roman est redevenu soupçonneux il me semble. Peut-être que l'histoire bégaie. J'ai encore la naïveté d'y être très attaché, mais en ce moment j'ai envie de faire autre chose; est-ce qu'à mon tour j'entre dans cette ère du soupçon ${ }^{4}$ ?

Prudemment modalisé, le propos n'en pose pas moins avec clarté, au début du XXI ${ }^{e}$ siècle, un retour du soupçon pesant sur le genre romanesque, ce qui, en bonne logique, suppose rétrospectivement sa dissipation momentanée au cours de la période qui a précédé...

Qu'on la partage intégralement, ou pour partie seulement, l'appréciation de Jean Echenoz se révèle à plus d'un titre digne d'intérêt. Tout

2. Aaron Kibedi Varga, «Le récit post-moderne », Littérature, n 77 , février 1990, p. 16 et passim.

3. «Entretien avec Jean Echenoz » (propos recueillis par Sidonie Loubry-Carette), Roman 20-50, n 38, décembre 2004, p. 5. Ce numéro de la revue contient un « dossier critique » consacré à "Jean Echenoz, Les Grandes Blondes, Un an et Je m'en vais », dirigé par Sidonie Loubry-Carette.

4. Ibid. 
d'abord, en ce qui concerne son œuvre personnelle, l'écrivain fait preuve d'une indéniable lucidité : de Nous trois à Au piano, la pratique du détournement hypogénérique, qui constituait sa «marque de fabrique » depuis sa première publication, s'est considérablement édulcorée en même temps que complexifiée; et l'on sait que, peu après, Ravel, paru en 2006, marque le début d'une série de « biofictions », qui à ce jour comprend deux autres volumes, Courir et Des éclairs ${ }^{5}$. En outre, il fait à la fois preuve d'humilité et d'une louable prudence en n'hypostasiant pas sa relation idiosyncrasique au roman en norme globalisante - quand bien même nombre d'auteurs

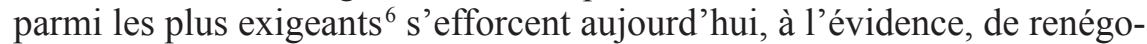
cier leur relation à la fiction romanesque, de plus en plus fréquemment hybridée de récit personnel et/ou factuel. Ensuite, l'hypothèse d'un bégaiement de l'histoire mérite d'être prise au sérieux : qu'il s'agisse de théorie de la littérature ou d'histoire littéraire, les exemples de ce que l'on pourrait de façon légèrement différente présenter comme autant de « retours de balancier » sont légion ${ }^{7}$. Enfin, on aura relevé l'emploi du substantif « naïveté » pour spécifier l'attachement durable de l'écrivain à la forme roman, en dépit du soupçon renaissant qu'il évoque. C'est précisément ce dernier point qui selon moi fait "problème ", et mérite par là même qu'on s'y attarde. « Naïveté » de Jean Echenoz? Vraiment? Appliqué à cet auteur, le terme semble à ce point impropre que forte est la tentation d'y lire l'indice d'une modestie vraie ou fausse, voire d'une coquetterie d'écrivain. Dans la formule résonne certes l'écho des préventions d'un modernisme qui, de Paul Valéry et des surréalistes au Nouveau Roman et à Tel Quel, s'est toujours défié des séductions jugées pernicieuses d'un genre fondé sur l'arbitraire de la fabula ${ }^{8}$. Mais, précisément, même à l'époque où, selon ses dires, la pratique romanesque était à ses yeux exempte de soupçon, Jean Echenoz ne s'est jamais livré à un usage naïf du genre, tout au contraire, puisque le « procès romanesque » s'est toujours doublé chez lui d'un « procès $d u$ romanesque $^{9} \gg$. Ainsi, chez Jean Echenoz, mais aussi de façon beaucoup plus générale, la multiplication de tours et détours narratifs favorise la

5. Nous trois (1992), Au piano (2003), Ravel (2006), Courir (2008) et Des éclairs (2010) ont tous été publiés aux éditions de Minuit.

6. Pierre Guyotat, Raymond Federman, Alain Fleischer, Pierre Michon, Régis Jauffret, Emmanuel Carrère, pour n'en citer que quelques-uns.

7. Sur ces questions, voir Antoine Compagnon, Les Cinq Paradoxes de la modernité, Paris, Le Seuil, 1990, et Le Démon de la théorie, littérature et sens commun, Paris, Le Seuil, 1998.

8. Au sens qu'Umberto Eco donne à ce terme dans Lector in fabula, ou la coopération interprétative dans les textes narratifs [1979], Paris, Grasset, 1985.

9. Bruno Blanckeman, Les Récits indécidables, Jean Echenoz, Hervé Guibert, Pascal Quignard, Villeneuve d'Ascq, Presses universitaires du Septentrion, coll. «Perspectives », 2000, chap. II (« Jean Echenoz, portrait de l'écrivain en simulateur»), p. 27. Dans la suite de cet article, de nombreux renvois seront faits à cet ouvrage comme aux autres travaux de Bruno Blanckeman sur l'œuvre de Jean Echenoz. 
conciliation de fabulae revigorées et d'une lucidité (auto-)critique des plus aiguës. On distinguera donc avec soin la naïveté de l'attachement au roman et la naïveté de la pratique romanesque, l'absence de la seconde incitant à fortement nuancer l'existence de la première.

Selon les spécialistes de l'œuvre, l'acuité du regard critique echenozien s'exercerait selon trois principales lignes de force, au demeurant étroitement intriquées. Ainsi, en particulier, Bruno Blanckeman y identifie-t-il la présence sous-jacente d'un projet tripartite, s'actualisant en un « travail romanesque de modélisation esthétique du récit », un « travail romanesque de modalisation ethnographique », et un « travail romanesque de médiation ontologique ${ }^{10}$ ». Le premier pôle correspondrait, sur fond d' " esthétique de la désinvolture ${ }^{11}$ », à un jeu sur/avec les genres et les sous-genres, l'histoire des formes, bref la mémoire littéraire. D'où, aux antipodes de tout dogmatisme, une forme discrète et volontiers ironique de « théorisation » du roman, entreprise depuis son sein même - dimension métatextuelle portant sur des objets variés, et que ses modalités mêmes d'apparition rendent plus ou moins aisément perceptible. Le deuxième pôle se déduirait quant à lui d'une constante de la fiction romanesque echenozienne, toujours en prise sur une conscience de l'Histoire en cours. Les huit premiers romans édifient ainsi des univers contrefactuels ${ }^{12}$, possédant la particularité commune de renvoyer au nôtre dans ce qu'il a de plus contemporain - cette attention aux realia du jour s'actualisant dans le relevé pointilliste de détails, faits, phénomènes et comportements potentiellement révélateurs de l'état présent de la société, de ses métamorphoses, de ses aspects inédits, partant de son devenir. Enfin, le troisième pôle procéderait d'une attention portée aux personnages de ces fictions qui, certes sur un mode dérisoire, n'en constituent pas moins autant d'échantillons de l'humanité actuelle, en proie à des mutations similaires à celles que connaît la société d'aujourd'hui. Les romans de Jean Echenoz proposeraient ainsi de surcroît une discrète radiographie de «l'être au présent », sur le double plan des identités collectives et individuelles.

Sans doute pourrait-on débattre à l'envi de l'importance respective ou du poids relatif de ces trois lignes de force dans l'œuvre, voire contester la présence d'une réflexion - quand bien même « en acte »- de type ethnologique et ontologique dans des romans réputés pour leur formalisme, voire suspects selon certains de légèreté. Peuvent notamment y inciter certaines des déclarations de Jean Echenoz, qui en la matière ne fait pas mystère de ses priorités. Ainsi, à la question : "Est-ce que vous cherchez, dans vos romans, à faire passer des messages? », répond-il sans ambages :

10. Bruno Blanckeman, « En théorie, “je m’en vais” », Roman 20-50, n³8, décembre 2004, p. 78 .

11. Bruno Blanckeman, Les Récits indécidables, op. cit., p. 27 et passim.

12. Thomas Pavel, Univers de la fiction [Fictional Worlds, 1986], Paris, Le Seuil, 1988. 
Je ne crois pas que ce soit ma vocation première. Ma vocation, mon désir, c'est de fabriquer de petites machines. De fabriquer des romans comme des machines. Pour moi, la mécanique et l'esthétique sont plus importants que le message ${ }^{13}$.

Mais que l'on ne s'y méprenne pas : une telle revendication du primat de l'esthétique, de la technique sur les autres composantes de la pratique romanesque, est très loin d'équivaloir à une proclamation de formalisme « gratuit ». De fait, ailleurs, Jean Echenoz ne nie pas la possibilité d'une influence de ses études de sociologie sur son travail d'écriture ${ }^{14}$, et revendique " un souci $[. .$.$] de rendre compte de l'état du monde, même si ce$ n'est pas [son] désir premier ${ }^{15} »$. Or nous savons, au moins depuis Roland Barthes ${ }^{16}$, qu'un texte littéraire peut être défini comme un tissu de codes multiples, dont l'entrelacement génère une pluralité sémantique. Que les priorités conscientes et déclarées de Jean Echenoz se portent sur des questions de «mécanique narrative » n'empêche donc en rien - tout au contraire - son œuvre de constituer un exemple de « formalisme plein, de type spéculatif ${ }^{17}$ » et aux objets multiples. Au temps pour la présumée superficialité d'une œuvre dont le regard attentif a tôt fait de détecter la richesse du « feuilleté ».

Cela posé, dans l'espoir de mieux cerner les tenants et les aboutissants de ce « retour d'affection pour le roman » et le romanesque dans l'œuvre echenozienne, je me propose, non pas d'en offrir une vue cavalière dans sa globalité, mais d'en scruter un « fragment », sous la forme d'une microlecture du début du chapitre 28 de Je m'en vais ${ }^{18}$. L'entreprise pourrait de prime abord passer pour une gageure, tant l'esthétique echenozienne ne paraît donner sa pleine mesure qu'envisagée à l'échelle supérieure du tissu unissant deux (Un $a n^{19}$ et Je m'en vais), trois (ajoutons-y Les Grandes

13. « Dans l'atelier de l'écrivain » (entretien avec Jean Echenoz, réalisé le 28 octobre 1999, pour les éditions Bréal, par Geneviève Winter, Pascaline Griton et Emmanuel Barthélémy; publié dans Français seconde, Paris, Bréal, 2000, puis repris dans la réédition de Je m'en vais dans la collection « Minuit Double », que je cite), p. 242.

14. «Entretien avec Jean Echenoz », op. cit., p. 11-12.

15. Ibid., p. 11.

16. Roland Barthes, $S / Z$, Paris, Le Seuil, 1970.

17. Bruno Blanckeman, «"... un petit rire de gorge...": le jeu mode d'emploi dans Les Gommes et La Jalousie », dans Frank Wagner et Francine Dugast-Portes (dir.), Lectures de Robbe-Grillet, Rennes, Presses universitaires de Rennes, 2010, p. 152.

18. Principalement de «Personnellement, je commence à en avoir un peu assez, de Baumgartner » (p. 170) à « avant de s'effondrer, souvent latéralement et perpendiculaire à ses pattes raidies » (p. 171).

19. Jean Echenoz, Un an, Paris, Minuit, 1997. Sur les liens qui unissent Un an et Je m'en vais, on pourra se reporter à Dominique Viart, «Les esthétiques démenties : réalisme et minimalisme d'Un an à Je m'en vais », Roman 20-50, no 38, décembre 2004, p. 15-26; Christine Jérusalem, «L'occupation des sols fictionnels dans les romans de Jean Echenoz: correspondances et allers-retours » (ibid., p. 27-35); Laurent Demanze, « Lecture d'une 
Blondes $^{20}$ ) voire $n$ textes (l'œuvre). Même si l'on accepte d'en rabattre quelque peu sur ce plan, en se concentrant sur un seul roman, nombre de difficultés subsistent, en raison de la prépondérance qui y revient à l'intrigue, au sens fort du terme, tel que nous l'a légué une tradition de pensée remontant à Aristote. Ainsi dans Je m'en vais, où l'alternance contrapunctique, jusqu'à leur convergence finale, de deux lignes d'histoire portées par des temps distincts (passé pour l'une, celle de Ferrer; présent pour l'autre, celle de Delahaye alias Baumgartner) semble constituer un défi aux tentatives d'autonomisation d'une séquence donnée.

Mais si un tel geste critique, pour problématique qu'il soit, se révèle malgré tout possible, c'est que l'œuvre de Jean Echenoz procède assez largement d'une dynamique paradoxale, entre continuité et discontinuité, cohésion et atomisation. Dans la construction de ces «machines célibataires $^{21} \gg$ que sont ses romans, Jean Echenoz préfère à la ligne droite - plus court chemin d'un point à un autre - l'oblique et les chemins de traverse, conformément à l'essence même du genre : célibataire ou non, le roman est aussi, peut-être surtout, une machine à différer l'information. Les deux premières pages du chapitre 28 de Je m'en vais offrent ainsi une excellente illustration de ce que faire narration buissonnière veut dire. Mais, pour les raisons susdites, avant de s'atteler à l'étude de cette séquence, sans craindre parfois d'en excéder les « frontières », il parait nécessaire de proposer une vision d'ensemble de l'esthétique à l'œuvre dans ce roman.

\section{Mémoire de la littérature / des littératures}

Conformément à la hiérarchie établie par l'auteur, ce qui accapare d'emblée l'attention à la lecture de son huitième roman est sans contredit l'intense travail de modélisation esthétique auquel il s'y livre - activité qui présuppose la vive conscience de s'inscrire dans un champ déterminé, caractérisable de surcroît par son historicité. De fait, à certains égards, Je m'en vais peut être considéré comme un Mémorial (ambigu) dressé à la gloire de la littérature, conçue simultanément comme patrimoine et comme répertoire de formes. À l'examen, tous les procédés que Gérard Genette rassemble sous les dénominations de «transcendance textuelle du texte » ou de « transtextualité22 » y convergent à des fins d'édification de ce «monument» dont l'ambiguïté axiologique - antidote idéal à tout dogmatisme pontifiant - procède en premier lieu de l'irrévérence joueuse qui paraît présider à l'entreprise.

séquence » (ibid., p. 37-43); et Frank Wagner, « Lire l'entre-deux », La Lecture littéraire, $\mathrm{n}^{\circ}$ 5-6, avril 2002, p. 125-137.

20. Jean Echenoz, Les Grandes Blondes, Paris, Minuit, 1995.

21. «J'essaie de construire mes fictions comme on construit des machines plus ou moins célibataires » («Entretien avec Jean Echenoz », op. cit., p. 11).

22. Gérard Genette, Palimpsestes, la littérature au second degré, Paris, Le Seuil, 1982, p. 7. 


\section{Architextualité}

De l'« architextualité ${ }^{23}$ » participe ainsi le traitement du genre, ou plutôt, en l'occurrence, d'une diversité de sous-genres. Si cette dimension double de l'écriture apparaît moins évidente qu'à l'époque de $\mathrm{Lac}^{24}$, par exemple, tout entier fondé sur une dynamique d'emprunts distanciés au seul roman d'espionnage ${ }^{25}$, c'est que Je m'en vais repose, de façon légèrement différente, sur une pratique de « collage » hypogénérique. En effet, Jean Echenoz y orchestre la rencontre d'au moins deux sous-genres, fréquemment perçus, à des degrés divers, et à plus ou moins juste titre, comme paralittéraires ${ }^{26}:$ du roman d'aventures participe le récit inaugural de l'expédition arctique de Félix Ferrer; du roman noir celui, conséquent, de diverses manigances crapuleuses (vol, meurtre) et de leurs répercussions (enquête, traque, règlement de comptes). La convocation de ces horizons d'attente notoires, par la réactivation de leurs figures imposées respectives, contribue à sécuriser le lecteur en le plaçant en terrain de connaissance, ce qui facilite considérablement, et à relativement peu de frais, son immersion fictionnelle $^{27}$. En ce sens, il y a indéniablement retour du récit, ou plutôt retour au récit, si l'on entend désigner par là une façon de renouer avec les potentielles structures d'emprise de fabulae d'autant plus efficaces qu'elles s'inscrivent dans des moules ou matrices éprouvés, et par là même aisément identifiables.

Mais, loin de se contenter de puiser dans ce réservoir de stéréotypes, Jean Echenoz paraît surtout les convoquer dans le dessein de les révoquer, au moins pour partie, en attirant implicitement l'attention lectorale sur leur degré d'usure, comme sur leur inadéquation au monde contemporain qu'il s'agit ici, quand bien même de façon seconde, de dire. De cette esthétique distanciée et/donc distanciatrice relève le collage ou le télescopage précédemment évoqué, qui aboutit à un texte génériquement hybride : le périple polaire de Ferrer n'a qu'un temps; lui succède la traque de l'associé indélicat (Delahaye/Baumgartner), à son tour délaissée in fine - le roman s'achevant, comme il avait débuté, sur l'évocation des difficultés amoureuses de son antihéros. À y regarder de plus près, au fil de sa diachronie, Je m'en vais témoigne donc du jeu d'une triple dynamique hypogénérique, puisque du nord au sud et de femme en femme, le sous-genre du récit sentimental y tient lieu de basse continue. À l'échelle du texte, les frictions de ces divers codes, a priori non homogènes, peuvent contribuer à les désigner comme tels, et par là même à les évider au moins partiellement de leur stéréotypie.

23. Ibid. Genette avait antérieurement formalisé cette notion dans Introduction à l'architexte, Paris, Le Seuil, 1979.

24. Jean Echenoz, Lac, Paris, Minuit, 1989.

25. Même si, dès cette époque, le récit d'espionnage était « concurrencé » par le récit sentimental, via l'évocation des amours de Franck Chopin et Suzy Clair.

26. Sur la notion de paralittérature, et les problèmes qu'elle pose, voir Daniel Couégnas, Introduction à la paralittérature, Paris, Le Seuil, 1992.

27. Jean-Marie Schaeffer, Pourquoi la fiction?, Paris, Le Seuil, 1999. 
D'autant que lesdits codes sont soumis à un traitement des plus anomiques, tant sur le plan macrostructurel - le travail d'une narration virtuose et volontiers exhibitionniste - que sur le plan microstructurel - le travail de l'écriture, dont les aspérités révèlent à chaque page la nature de medium ambigu, car enclin à la polysémie et à l'opacité.

Bref, ces ressources convergentes permettent à Jean Echenoz de proposer une «vraie-fausse » littérature de genre, en littérarisant, voire en « hyperlittérarisant » des éléments (diégétiques aussi bien que structurels) d'ordinaires réputés paralittéraires - geste de recyclage typiquement postmoderne consistant à faire du neuf avec du vieux. Toutefois, que l'on ne s'empresse pas de conclure, de la part de Jean Echenoz, à une condescendance élitiste voire élitaire à l'égard des traditions hypogénériques concernées : la réélaboration à laquelle il les soumet témoigne selon moi d'une relation beaucoup plus ambivalente, entre phase et déphasage, accord et écart. Ce qui est ici à l'œuvre tient en effet du double jeu, car ces textes stratifiés offrent simultanément à leurs lecteurs la jouissance esthétique dispensée par des histoires captivantes et la jouissance stratégique autorisée par la dénudation biaise des rouages qui, à tous les niveaux, les sous-tendent.

Dans le chapitre 28 de Je m'en vais, dont le début peut faire figure d'excursus ou de dérive narrative, l'esthétique du double fond qui vient d'être évoquée se cristallise dans la figure de « Baumgartner », pseudonyme adopté par Delahaye, ancien associé de Félix Ferrer, pour se soustraire aux recherches, après qu'il a dérobé les objets d'art inuit rapportés du pôle Nord par son employeur. À un premier niveau de lecture, l'emprunt à l'horizon d'attente du roman noir affecte l'ancrage diégétique et les événements (ou plutôt l'absence d'événements) qui y surviennent : si ce chapitre se déroule dans le Sud-Ouest de la France, si le personnage que nous y suivons mène une existence solitaire, morne et répétitive, c'est précisément parce que, par suite de son forfait, il se trouve « en cavale ». Mais le choix même du pseudonyme - qui relève des prérogatives du scripteur - favorise la mise en place d'un second niveau de lecture, en porte-à-faux avec le premier : les lecteurs avertis, c'est-à-dire ceux dont le fonds encyclopédique personnel contient cette donnée, auront remarqué la forte proximité phonétique, d'ailleurs ponctuellement soulignée par le texte ${ }^{28}$, entre «Baumgartner » et... « Baumgarten », philosophe allemand du XVIII ${ }^{\mathrm{e}}$ siècle, que 1'on considère généralement comme l'inventeur du néologisme « esthétique ». Le traitement de l'onomastique favorise ainsi la rencontre improbable, dans une station balnéaire du Sud-Ouest, du roman noir et de la théorie de l'art. Forte est dès lors la tentation d'y lire une métaphorisation ironique de l'activité d'écriture foncièrement hybride à laquelle s'adonne Jean Echenoz.

28. « Je viens pour le studio du cinquième qui est à louer, a-t-il dit, c'est moi qui vous ai téléphoné lundi pour visiter. Ah oui, s'est souvenue la gardienne, au nom de Baumgarten? Tner, a corrigé l'homme, Baumgartner. » (Jean Echenoz, Je m'en vais, op. cit., p. 71.) 


\section{Intertextualité}

Mais, dans Je m'en vais, la mémoire de la littérature est très loin de se limiter à ce jeu architextuel avec des genres ou sous-genres codifiés, renouvelés par hybridation (ou greffe) et subversion (ou détournement). Y contribue également la mise en œuvre des ressources de l'intertextualité dont, précisément, la dernière page du chapitre 28 propose un exemple des plus savoureux : « Il [Baumgartner] connaît la mélancolie des restauroutes, les réveils acides des chambres d'hôtels pas encore chauffées, l'étourdissement des zones rurales et des chantiers, l'amertume des sympathies impossibles ${ }^{29} »$; qui démarque ironiquement un célèbre passage de la fin de L'Éducation sentimentale : « Il [Frédéric Moreau] connut la mélancolie des paquebots, les froids réveils sous la tente, l'étourdissement des paysages et des ruines, l'amertume des sympathies interrompues ${ }^{30}$. \ Cette ponctuelle activité de réécriture ${ }^{31}$ revêt les allures d'un clin d'œil ludique, fondé sur l'introduction d'un faisceau de différences entre le « texte-source » et le « texte-cible » : substitution du présent de l'indicatif au passé simple, des réalités de notre époque à celles du XIX ${ }^{\mathrm{e}}$ siècle - corroborant la dimension discrètement ethnographique du roman selon Jean Echenoz. Par-delà le procédé même de l'interpolation, la suture avec le corps du récit se révèle également significative. Â la différence de Frédéric Moreau, de ses pérégrinations Delahaye alias Baumgartner ne tire en effet aucune leçon existentielle : « Cela dure encore à peu près deux semaines au terme desquelles, vers la mi-octobre, Baumgartner s'aperçoit enfin qu'il est suivi ${ }^{32}$. » $\mathrm{Ce}$ que l'on pourrait nommer la « piste Flaubert» tourne court; s'ensuit un retour à la « paralittérature » par la mention de la filature, qui constitue un archétype structurel du roman noir. On notera en outre la plurivocité de l'ironie echenozienne, lisible d'une part dans la «modernisation » du passage emprunté à Flaubert, d'autre part dans la modalisation du discours narratorial par l'adverbe « enfin », qui révèle la position surplombante de l'instance narrative à l'égard du personnage.

Quelles peuvent donc être les fonctions d'un tel traitement de l'intertextualité, dont Jean Echenoz est coutumier ${ }^{33}$ ? D'aucuns y verront un simple clin d'œil ludique :

29. Ibid., p. 176.

30. Gustave Flaubert, L'Éducation sentimentale [1869], partie III, chap. vI (Paris, Gallimard, 1995, coll. « Folio », p. 450 pour l'édition utilisée).

31. Qui, dans la terminologie genettienne (Palimpsestes, op. cit.), relève donc de l'hypertextualité. Mais, dans la mesure où le phénomène de réécriture est étroitement circonscrit, il paraît préférable de parler, à l'échelle du roman, d'intertextualité. En définitive, la coprésence de ces ressources n'a rien de surprenant, qui concourent de conserve à la dimension double de l'écriture echenozienne.

32. Jean Echenoz, Je m'en vais, op. cit., p. 176.

33. Dans Je m'en vais, on mentionnera par exemple cet autre passage (p. 51), qui « recycle» le célèbre épisode rabelaisien (Quart Livre, chap. XV-XVI) des «paroles gelées » : «Ferrer 
- à la littérature, citée à comparaître en la personne de l'une de ses figures emblématiques;

- à l'un des auteurs appréciés de Jean Echenoz, dont le goût pour l'œuvre de Flaubert, précurseur de la modernité et maître ès ironie, est bien connu.

Mais, par-delà l'amusement inhérent à ce procédé, la présence ponctuelle dans ses romans d'extraits, ni attribués ni même démarqués, de textes littéraires, lui permet d'y ménager une sorte de double fond où résonne l'écho de la littérature des siècles passés - ce qui contribue, quand bien même avec discrétion, à conserver active la mémoire de certaines œuvres majeures. En jouant de cette esthétique de la farcissure, Jean Echenoz assume ainsi un rôle de passeur, sans pour autant risquer d'empoisser ses écrits aux accents prudhommesques qui accompagnent trop souvent les revendications patrimoniales. En outre, comme l'a montré l'exemple précédent, ces raccords textuels autorisent la dialectisation oblique d'une esthétique hybride, ne craignant pas d'apparier des horizons d'attente non seulement contrastés, mais situés aux extrémités de l'échelle de valeurs que reconnaît l'institution littéraire. Enfin, si clin d'œil il y a, il s'adresse aussi aux lecteurs, dont le « bagage » culturel leur permet d'identifier l'intertexte interpolé, le sentiment de partage qui en résulte favorisant alors de façon très efficace l'établissement d'une relation de connivence.

Encore ces « emprunts » ponctuels ne représentent-ils qu'un cas particulier de cette dynamique transtextuelle constitutivement relationnelle que l'on baptise « intertextualité ». Et c'est de façon beaucoup plus générale, sans qu'il soit toujours possible d'identifier avec assurance la présence sousjacente d'un texte ou d'un auteur précis, que dans ses romans Jean Echenoz pratique cette littérature « au carré ». Parmi d'autres exemples, Je m'en vais en recèle un échantillon selon moi particulièrement significatif :

\footnotetext{
À Biarritz, ce dimanche, près du phare, Baumgartner voit un jeune homme s'aventurer au plus près de l'océan, au bord extrême d'une avancée rocheuse, risquant de se faire absolument tremper par les bourrasques d'écume nerveuse qu'il esquive avec un déhanchement de torero. C'est d'ailleurs en termes taurins qu'il commente la puissance des vagues successives, salue $(\mathrm{Ole})$ une explosion spécialement scénique, laisse venir (Mira mira mira) et gonfler (Toro toro) une vague prometteuse et grondante (Torito bueno) - tous encouragements, appels et citations que dans l'arène on adresse aux fauves. Puis après que la vague a sauvagement rué en tous sens, s'est disloquée en déflagrant, quand ce monstre en eau vient se coucher
}

essaya au début d'échanger quelques propos avec ses guides, surtout Angoutretok qui possédait un peu d'anglais, Napaseekadlak ne s'exprimant que par sourires. Mais les paroles, une fois émises, sonnaient trop brièvement avant de se solidifier : comme elles restaient un instant gelées au milieu de l'air, il suffisait ensuite de tendre une main pour qu'y retombent, en vrac, des mots qui venaient doucement fondre entre vos doigts avant de s'éteindre en chuchotant. » 
et mourir à ses pieds, le jeune homme, bras tendu et main levée comme pour immobiliser le temps, lui adresse le geste des matadors dans l'intervalle, parfois un peu long, où la bête estoquée demeure dressée pendant que la vie lui échappe avant de s'effondrer, souvent latéralement et perpendiculaire à ses pattes raidies ${ }^{34}$.

En apparence narrativement incongrue, cette courte scène, caractéristique de ces poussées de « romanesque hors champ ${ }^{35}$ » que Jean Echenoz se plait à faire bourgeonner sur le corps de ses romans, peut pourtant être remotivée, dès lors que l'on prête attention aux échos intertextuels susceptibles de s'y faire entendre. Dans cette perspective de lecture, la figure de ce jeune homme juché sur des rochers, qui défie les éléments déchainés assimilés à quelque animal monstrueux, peut être considérée comme la réactivation iconoclaste d'un archétype romantique - de Chateaubriand à Hugo, les exemples ne manquent pas -, progressivement commué au fil du temps en stéréotype. En outre, la mémoire de la littérature est en l'occurrence aussi mémoire des formes littéraires : on aura remarqué la focalisation interne inaugurale, opérée par un narrateur au demeurant omniscient, sur le personnage de Baumgartner, ainsi constitué en un foyer perceptif par le truchement duquel nous sont communiquées diverses informations visuelles et auditives. Si l'on y reconnaît un nouveau clin d'œil, aux habitudes narratives en vigueur dans le roman du XIX $X^{e}$ siècle cette fois, il est alors possible de conclure à une rencontre ou fusion des stéréotypes thématique et technique, le traitement loufoque de la scène contribuant dans le temps même de son déploiement à désigner comme telle cette stéréotypie redoublée, ce qui permet de la mettre ainsi à distance, donc de la désamorcer. D'autant qu'en termes pragmatiques, un troisième type de ressource semble pouvoir renforcer les effets des deux procédés déjà identifiés : sur le plan rhétorique, l'assimilation, toute la séquence durant, des flots déchainés à un animal furieux (ici, un taureau) relève de la métaphore filée. Cette métaphore convenue jusqu'au cliché est :

- comme « déléguée » à l'un des personnages de la diégèse - le jeune homme, dont le comportement révèle par induction le transfert analogique qu'il opère;

- prise au pied de la lettre - puisque la pantomime induit la « présence » du métaphoriquant;

- dévidée jusqu'à son terme narratif logique - le retrait de la vague équivalant à la mort du taureau.

À l'examen, ce micro-texte procède donc d'une matrice à la fois intertextuelle (l'horizon d'attente romantique), narrative (la délégation stéréotypée du point de vue comme préambule au déploiement d'une séquence descriptive) et stylistique (le procédé de la métaphore filée). Aussi

34. Ibid., p. 171.

35. Bruno Blanckeman, "En théorie, “je m'en vais" », op. cit., p. 80. 
ne paraît-il pas excessif de parler ici d'une forme d'auto-engendrement ludique, dont les effets oscillent entre vraisemblance et fantaisie potentiellement déréalisante.

De la vraisemblance participe jusqu'à un certain point la justification de ces éléments diégétiques par le chronotope : le Sud-Ouest de la France est terre de tradition tauromachique, proximité de cette région avec la frontière espagnole oblige; de sorte que le recours ponctuel à cet idiome se trouve pour partie motivé. Mais cette vraisemblance de façade est largement débordée par les effets pervers ou parasitaires des procédés de textualisation mis en œuvre. Ainsi des modalités mêmes de l'inscription des notations en espagnol, scansion sonore qui rythme le déroulement de la scène, et dont le systématisme génère un effet comique. La distanciation procède en fait surtout de la consignation de ces incidentes en italiques et entre parenthèses, ce qui contribue à désigner ces emprunts au lexique de la tauromachie, en version originale, comme autant de corps (linguistiques) étrangers, comme tels imparfaitement assimilables. Voilà qui contribue en outre, parmi d'autres ressources aux effets convergents, à conférer à la voix narratoriale une dimension « ventriloqu[é] $\mathrm{e}^{36}$ ».

Par cette interpolation de bribes d'idiome vernaculaire, la texture même du passage témoigne donc de la propension du scripteur à la fantaisie, qui redouble hors-diégèse la caractérisation du personnage intradiégétique, « doux dingue » ou « illuminé » inoffensif ne mettant en danger tout relatif que sa propre personne. Serait-ce forcer le sens que d'affirmer qu'il prend les vagues pour des taureaux comme un autre, avant lui, prit des moulins à vent pour des géants? Après tout, les plus célèbres des châteaux en Espagne sont ceux de la fiction, dont la figure du Quichotte constitue par excellence l'emblème. Le personnage du jeune homme, visiblement quelque peu « amok »-le roman ne précise hélas pas s'il vient de Cadix... -, favoriserait ainsi la corruption, j'ose à peine dire la pollution, de l'intertexte romantique précédemment identifié par un clin d'œil à ce père du roman moderne et de l'anti-roman qu'est Cervantès. En outre, brochant sur le tout, il importe d'insister de nouveau sur la fantaisie du narrateur, qui se détourne du droit chemin pour rapporter un tel épisode ne contribuant en rien à la progression de l'histoire racontée, au point que d'aucuns y verraient peut-être un kyste disgracieux sur le corps de la narration. À vrai dire, de telles excroissances ne sont qu'en apparence immotivées; d'une part, comme nous venons de le voir, parce qu'elles contribuent à la stratification du roman echenozien, en le dotant d'un double ou triple fond intertextuel, d'autant plus aisément perceptible que le déroulement de la fabula y connaît un temps mort; d'autre part parce que ces excursus constituent un emplacement privilégié pour le travail de modalisation ethnographique et/ou de médiation ontologique.

36. Bruno Blanckeman, « Jean Echenoz ou le récit ventriloque », Cahiers de narratologie, $\mathrm{n}^{\circ}$ 10, La Voix narrative, 2001, vol. 1, p. 213 et passim. 
De telles « vignettes » favorisent en effet l'esquisse d'une cartographie du monde actuel et de ses habitants : ici, un Quichotte des temps postmodernes exhibant sa mythomanie sous 1'œil bovin des badauds attroupés, sur fond de désœuvrement dominical et provincial... Enfin, en dernier ressort, et puisque cette instance est de nos jours ressuscitée, la fantaisie qui coiffe toutes les autres est celle de l'auteur, qui en amont crée ce narrateur, prêt à tous les vagabondages, à toutes les dérives. Ce n'est d'ailleurs pas le moindre charme de ses fictions que la façon dont Jean Echenoz s'y écarte ponctuellement d'intrigues à la trame par ailleurs extrêmement serrée. $\mathrm{Ou}$ de la divagation considérée comme un des Beaux-Arts.

\section{Métatextuel ${ }^{37}$}

L'étude du bref excursus de la page 171 de Je m'en vais a donc permis d'établir la forte cohérence de l'écriture echenozienne, dont les composantes intertextuelles, stylistiques et narratives semblent concourir à la production d'un même effet. Approfondir l'examen du troisième de ces paramètres peut se révéler précieux pour mieux cerner l'esthétique d'un romancier aussi féru, selon ses propres termes, de "mécanique ». Ainsi, dans l'exemple qui vient d'être analysé, avec le choix d'une scène conduite en focalisation interne pour un bénéfice narratif à peu près nul, le romancier frôle la parodie, puisque a contrario, dans le roman « canonique », le procédé était d'ordinaire employé pour révéler des éléments diégétiques de première importance, et ainsi dynamiser et dramatiser la progression de 1'histoire racontée. Pour sa part, Jean Echenoz détourne ce topos narratif de sa fonction première, pour n'en conserver qu'une forme qu'il évide de son sens endoxal - ce qui lui permet de lui assigner ironiquement de tout autres fonctionnalités.

Or, cette stratégie subversive préside également, de façon à la fois plus fréquente, plus visible, plus générale et plus décisive, au traitement de l'arbitraire inhérent à la conduite du récit fictionnel par un narrateur omniscient et omnipotent. Sur fond de très vive mémoire des formes romanesques, s'ensuit ainsi une mise en crise de l'autorité narrative ${ }^{38}$. Dans Je m'en vais,

37. À distinguer de «la métatextualité », définie par Gérard Genette comme « la relation [...] de "commentaire", qui unit un texte à un autre texte dont il parle» (Palimpsestes, op. cit., p. 10). Dans le sens que Bernard Magné donne à ce terme, «le métatextuel» renvoie différemment à l'ensemble des moyens dont dispose un texte «pour assurer dans son corps même la désignation de tout ou partie de ses mécanismes constitutifs » (Protée, vol. XIV, n 1-2, 1986, p. 77), en jouant tantôt des ressources dénotatives, tantôt des ressources connotatives de la langue. C'est donc de cette relation d'autocommentaire, interne au texte, qu'il sera ici question.

38. Sur cette question, voir notamment Frances Fortier et Andrée Mercier, « Savoir retenu et savoir manquant, quelques enjeux de la narration omnisciente dans le récit contemporain », dans Cahiers de narratologie, n' ${ }^{\circ}$ 10, vol. 1, 2001, p. 445-460. 
en particulier, se fait jour un traitement foncièrement irrévérencieux de la voix, qui prend à la fois pour cible les habitudes du roman « canonique » en la matière, et l'instance même qui rapporte les (més)aventures de Félix Ferrer, « narrateur d'opérette, qui rappelle la figure de l'écrivain omniscient comme les personnages de La Belle Hélène le modèle homérique ${ }^{39} »$. Jugeons-en sur pièces :

Ce spectacle [celui de l'océan furieux] hébète l'homme et le paralyse, il
peut indéfiniment le contempler sans se lasser, pas de raison de s'arrêter - le
feu aussi lui fait cet effet-là, la pluie quelquefois produit cet effet, l'inven-
taire des passants depuis une terrasse de bar peut le produire également ${ }^{40}$.

Cette observation incidente paraît tourner en dérision les abus qu'ont pu faire de l'omniscience narratoriale certains romanciers du passé (Hugo, Balzac, notamment), n'hésitant pas à assener sur des sujets variés, par le truchement d'un narrateur volontiers paternaliste, et fort d'un savoir en apparence illimité, autant de vérités générales, dont il ne restait aux lecteurs zélés qu'à convenir de la justesse avant d'en faire leur profit. C'est précisément cette dimension, rétrospectivement perçue aujourd'hui comme pesamment didactique, de certaines narrations « canoniques », que Jean Echenoz se réapproprie ici avec désinvolture et légèreté apparente. Ce traitement en fait duplice d'un procédé éprouvé transite tout d'abord par la reconduction des traits formels qui en assurent l'efficace : dissimulation (ponctuelle) de l'instance narrative derrière une impersonnalité de convention, ou si l'on préfère adoption d'une relation de personne apparemment hétérodiégétique; et surtout recours au présent gnomique, vecteur d'assertions de type dogmatique ne souffrant pas la contradiction. Mais ce moule formel n'est pas rigoureusement reproduit à l'identique, qui se voit altéré par une discrète oralisation du propos (« pas de raison de s'arrêter - le feu aussi lui fait cet effet-là »), par l'introduction de modalisateurs (« quelquefois », « peut»), et par le rapprochement trivial de la fin ("l'inventaire des passants depuis une terrasse de bar ») - qui constitue un nouvel îlot de modalisation ethnographique.

Quels qu'aient pu être, et quels que soient toujours, les indéniables mérites des romans du $\mathrm{XIX}^{\mathrm{e}}$ siècle, les considérables mutations épistémologiques survenues depuis lors ne permettent plus aujourd'hui à un romancier lucide et honnête de continuer à prendre à son compte la figure d'un narrateur à l'assurance inébranlable, dépositaire d'un savoir encyclopédique et dispensant à l'envi ses leçons - se dotant par là même d'un ethos des plus intimidants. Aussi peut-on comprendre que Jean Echenoz ne recoure au procédé qu'en le subvertissant partiellement par l'ironie : partiellement, car loin de le ruiner de façon irrémédiable, il lui conserve

39. Bruno Blanckeman, «Jean Echenoz ou le récit ventriloque », op. cit., p. 218.

40. Jean Echenoz, Je m'en vais, op. cit., p. 170. 
une bonne part de son efficacité. En effet, en dépit de la tonalité joueuse qui y préside, le contenu assertif n'est après tout pas faux : les phénomènes évoqués possèdent bel et bien ce pouvoir hypnotique identifié par le narrateur, qu'ils relèvent de la nature (les éléments que sont le feu et l'eau) ou de la " culture » (le défilé des passants observé depuis une terrasse de bar). Malgré sa trivialité et son potentiel comique, l'ultime notation correspond elle aussi à une forme de "vérité de dévoilement », et permet à Jean Echenoz, sans avoir l'air d'y toucher, de remplir l'un des rôles classiques du romancier. Mais, par les vertus de l'ironie et de l'humour, l'écrivain évite l'écueil du ton pontifiant, désormais irrecevable dans l'espace du roman, et ne nous fait partager son "savoir» sur le monde qu'à la faveur d'une opération de contrebande. Procédant de la sorte, il joue de nouveau triple jeu, puisqu'il parvient simultanément à adresser un clin d'œil à la pratique narrative de ses devanciers, à évider le procédé de sa stéréotypie, et à en préserver par là même la force pragmatique, subtilement renouvelée.

Souffler ainsi le chaud et le froid à propos de l'ethos et de l'étendue des connaissances de l'instance en charge du récit témoigne déjà d'un rapport ambivalent à la notion d'omniscience, que d'autres exemples permettent de confirmer, tout en attirant l'attention sur ses liens à la notion corollaire d'omnipotence - leur rencontre pouvant être subsumée, comme je l'ai déjà signalé, sous la dénomination d'arbitraire. En témoigne tout particulièrement l'ouverture du chapitre 28 :

Personnellement, je commence à en avoir un peu assez, de Baumgartner. Sa vie quotidienne est trop fastidieuse. À part vivre à l'hôtel, téléphoner tous les deux jours et visiter ce qui lui tombe sous la main, vraiment il ne fait pas grand-chose. Tout cela manque de ressort ${ }^{41}$.

Sur les diverses motivations d'un tel passage, Jean Echenoz s'est expliqué en ces termes :

un des personnages secondaires m'est tout à coup apparu d'un ennui total. J'aurais pu continuer de faire semblant, dissimuler cet ennui, le détourner... J'ai préféré le prendre à rebours et me plaindre de ce type qui, vraiment, ne faisait ni ne disait rien d'intéressant dans la vie. Dans ces cas-là, autant exprimer son désarroi, cela permet non seulement d'en finir, mais aussi tant qu'à faire d'agiter un peu la scène, de la rendre plus excitante, en me plaçant en porte-à-faux entre la position de l'auteur, celle du narrateur et celle du lecteur. Ce sont des détails, mais ces détails fonctionnent un peu comme des petites incongruités narratives - interpeller le lecteur, le prendre à partie - qui, en éclatant, rendent le mouvement d'ensemble plus tonique, et plus ludique ${ }^{42}$.

41. Ibid., p. 170.

42. Jean Echenoz, « Dans l'atelier de l'écrivain », op. cit., p. 244-245. 
De telles observations confirment, s'il en était besoin, la lucidité qui préside à l'emploi du procédé : que l'on réfléchisse à l'identification de l'instance narrative intruse ou aux effets qui en résultent, les deux paramètres témoignent d'une prise en compte ludique de la mémoire des formes littéraires, autant que d'une activité de «théorisation » biaise et enjouée. Sont tout d'abord confirmés les enseignements de la narratologie : «Tout récit est, explicitement ou non, "à la première personne", puisque son narrateur peut à tout moment se désigner lui-même par ledit pronom ${ }^{43}$. » $C^{\prime}$ 'est bien ce qui se produit dans l'extrait cité, où sa contestation ponctuelle et inopinée renvoie la relation hétérodiégétique environnante à son statut de convention artificielle, puisqu'il apparaît clairement à la faveur du commentaire intrus que la conduite du récit est assumée en sous-main par une instance de type homodiégétique. De plus, en termes de niveaux, puisqu'une instance extradiégétique ${ }^{44}$ fait irruption au sein de la diégèse, la frontière de la narration ou de la représentation elle-même se voit ainsi transgressée par cette métalepse narrative - procédé dont les propriétés dénudantes et déréalisantes ne sont plus à démontrer ${ }^{45}$. On se souvient qu'au fil du temps, les désignations du phénomène narratif ironiquement démarqué par Jean Echenoz ont varié : là où Georges Blin identifiait une « intrusion d'auteur ${ }^{46}$ ", Gérard Genette préférait pour sa part parler $\mathrm{d}^{\prime}$ " intrusion du narrateur ${ }^{47} »$. Écrivant quant à lui à l'époque de « la résurrection de l'auteur », Jean Echenoz parvient en quelque sorte à réconcilier ces deux positions théoriques, tout en œuvrant à leur dépassement :

\footnotetext{
il y a un jeu sur le « je ». Il peut y avoir plusieurs « je » : le « je » habituel, pronom du narrateur; le « je »-J.E., comme mes initiales, donc le « je » ou le jeu de l'auteur; mais au fond, c'est peut-être aussi un « je » tiers, on ne sait quel témoin incongru qui, passant par là, prendrait la parole, pourquoi $\operatorname{pas}^{48} \ldots$
}

De fait, à l'évidence, l'instance intruse qui assume le commentaire métatextuel sur les agissements répétitifs du personnage de Baumgartner renvoie à un narrateur de type auctorial, ce que Jean Echenoz souligne et dépasse en pointant l'homonymie du pronom et de ses initiales; de sorte que l'habituelle distinction entre instances narrative et littéraire perd de sa pertinence au point d'apparaître, du moins en l'occurrence, superflue. Quant à la troisième hypothèse, de prime abord aussi incongrue que le témoin

43. Gérard Genette, Nouveau discours du récit, Paris, Le Seuil, 1983, p. 65.

44. Ces diverses notions narratologiques renvoient bien évidemment, comme la plupart de celles qui sont mobilisées dans cet article, à Gérard Genette, Figures III, op. cit. et Nouveau discours du récit, op. cit.

45. Entre autres exemples, voir Gérard Genette, Métalepse, de la figure à la fiction, Paris, Le Seuil, 2003.

46. Georges Blin, Stendhal et les problèmes du roman, Paris, Corti, 1954, p. 312-322.

47. Gérard Genette, Figures III, op. cit., p. 244 et passim.

48. Jean Echenoz, « Dans l'atelier de l'écrivain », op. cit., p. 245. 
de passage qu'elle convoque, peut-être ne l'est-elle pas tant que cela. À l'époque actuelle en particulier, rien ne nous assure de l'homogénéité vocale du récit fictionnel : Jean Echenoz rejoint ainsi, à la faveur d'une apparente boutade, l'avis de ces théoriciens qui estiment que la narration fictionnelle peut être composite et fluctuante, car assumée par une pluralité d'instances se partageant, sans solution de continuité, la conduite du récit ${ }^{49}$.

Cela posé, qu'en est-il des fonctions et/ou des effets du procédé? Jean Echenoz signale lui-même que la « prise à partie » ou « l'interpellation du lecteur » permet simultanément de dynamiser la scène et de produire un effet ludique. En toute rigueur, l'extrait cité ne contient pas d'apostrophe stricto sensu, par conséquent n'édifie pas de narrataire invoqué. Mais il n'en est pas moins vrai que le flottement qui affecte les places et les rôles de l'auteur, du narrateur et du lecteur favorise une cooptation ludique de ce dernier - phénomène récurrent dans l'œuvre echenozienne, en raison en particulier des fluctuations pronominales, et plus encore de l'usage fortement ambivalent des pronoms « on » et « nous ${ }^{50} »$. Ici, tout se passe en effet comme si, par ce commentaire « parasite », Jean Echenoz s'efforçait, en prenant les devants, de désamorcer l'ennui probable d'un lecteur confronté aux agissements répétitifs du personnage, ce qui correspond à une forme d'intégration à la conduite même du récit du pôle de la réception - conformément à l'esthétique de la connivence déjà identifiée. L'essence bipolaire de la narration romanesque, reposant sur une interaction de ses instances émettrice et réceptrice, est ainsi dénudée et habilement mise au service de la captatio benevolentiae; car le récit s'en trouve bel et bien dynamisé, par l'introduction de jeu, au sens mécanique - provoquer des frictions entre les éléments constitutifs de la narration - aussi bien que ludique - susciter ainsi le rire ou le sourire du lecteur complice. La stratégie se révèle particulièrement adroite, car elle permet à l'écrivain de jouer la carte de la distanciation réflexive sans pour autant risquer de contrarier irrémédiablement les dispositions projectives des lecteurs. Par l'interpolation de tels commentaires métatextuels, le romancier signale ironiquement l'arbitraire de son pouvoir de fabrique, reposant sur la conjonction de l'omniscience (il « sait » tout puisqu'il lui est loisible de tout inventer à mesure) et de l'omnipotence (il a la haute main sur la conduite du récit, et barre sur son ou ses narrateurs). En effet, si l'on y réfléchit un instant, se plaindre d'un personnage fastidieux n'a pas réellement de sens ${ }^{51}$, puisqu'il suffit pour dissiper l'ennui de faire survenir une nouvelle péripétie. Lorsque cela se produit à la fin

49. Pour divers exemples de cette position, à certains égards « anti-narratologique », on se reportera à l'ouvrage de Sylvie Patron, Le Narrateur, introduction à la théorie narrative, Paris, Armand Colin, 2009, notamment au chapitre x, «La théorie du déplacement déictique » (p. 237-252).

50. Bruno Blanckeman, « Jean Echenoz ou le récit ventriloque », op. cit., p. 214.

51. Sauf, toutefois, à opérer une distinction stricte de l'auteur et du narrateur... à laquelle, en l'occurrence, n'incite pas la construction d'un narrateur auctorial. 
du chapitre ( «Baumgartner s'aperçoit enfin qu'il est suivi »), le lecteur attentif, aidé dans sa tâche par la présence de l'adverbe modalisateur, a tôt fait de reconsidérer le bien-fondé des récriminations auxquelles s'était tout d'abord laissé aller le narrateur - ce qui confère à la séquence des allures de démonstration par l'absurde. Mais ce qu'il y a ici de remarquable est que le romancier parvient à révéler l'étendue de ses pouvoirs comme l'artifice consubstantiel à la pratique romanesque sans y renoncer en rien, ni détourner irrévocablement le lecteur des séductions de l'histoire racontée.

Voilà donc amplement confirmées les observations antérieures, puisque nous sommes de nouveau confrontés à divers échos intertextuels (difficile en effet à qui les a lus de ne pas penser à Tristram Shandy et Jacques le fataliste) transitant par un jeu sur la mémoire des formes littéraires (la reprise du procédé de la métalepse "d'auteur»), à une esthétique de la désinvolture (les caprices du romancier l'autorisant à congédier la relation de personne - hétérodiégétique - qu'il a lui-même élue) et de la connivence (diverses stratégies convergeant à des fins de captation de la bienveillance lectorale), enfin à une entreprise de « théorisation » biaise (nous renseignant sur ce que signifie écrire et lire un récit de fiction aujourd'hui) se développant sans porter préjudice à une fabula captivante, servie de surcroît par une intrigue savamment concertée.

Qu'on y ajoute, à l'échelle du roman, la pratique antérieurement analysée de l'hybridation hypogénérique, et force sera de convenir que, dans Je m'en vais comme d'ailleurs dans le reste de son œuvre, Jean Echenoz réserve effectivement une place de choix au travail de modélisation esthétique.

\section{D'une pierre, trois coups}

Pour autant, comme l'a justement souligné Bruno Blanckeman, il serait injuste, sur la foi d'un tel constat, de porter un diagnostic de formalisme gratuit, tant le travail sur les formes apparaît en définitive indissociable d'une « radiographie » de la société contemporaine et de ses habitants, qui envisage de façon similaire le présent au prisme du passé - esquissant par là même les contours d'un futur problématique, mais déjà là à l'état embryonnaire. De cette discrète activité de modalisation ethnographique relèvent ainsi plusieurs des traits mentionnés lors de l'analyse des pages 170-171 de Je m'en vais:

- sur fond de désœuvrement dominical, l'attroupement des badauds face au spectacle de l'océan déchaîné;

- le rapprochement en apparence loufoque mais en définitive judicieux avec le pouvoir de fascination exercé par le défilé des passants sur l'observateur oisif qui les inventorie depuis la terrasse d'un bar;

- la pantomime du jeune homme, voué à la pratique du simulacre ludique dans un monde où les occasions d'affrontements plus décisifs tendent à se faire rares. 
On notera de plus que, via la convergence des ressources du simulacre et de la spectacularisation, le réel - du moins ce qui est donné pour tel dans la diégèse - fait l'objet d'une notable mise à distance, comme si s'estompaient les frontières du référent et de sa représentation ${ }^{52}$.

Cette tendance prononcée à la « sociologie amusante » culmine en outre à la page suivante :

Il [Baumgartner] aime mieux s'arrêter dans les villages, il y passe un moment au café-tabac sans parler à personne.

Il préfère y écouter les conversations des gens (quatre hommes inoccupés comparent leurs poids et le substituent au numéro de département français correspondant. Le plus maigre déclare donc la Meuse, l'à peu près normal revendique les Yvelines, l'assez épais admet frôler le Territoire de Belfort, le plus gros dépasse le Val-d'Oise), lire les affiches scotchées sur les miroirs (CONCOURS DE GROS LÉGUMES : 8 h-11 h, Inscription des légumes. 11 h-12 h 30, Opérations du Jury. 17 h, Remise des Prix et Vin d'honneur. Peuvent concourir : Poireau, Salade, Chou cabus, Chou de Milan, Choufleur, Chou rouge, Tomate, Melon, Potiron, Poivron, Courgette, Betterave rouge, Carotte rouge, Céleri-rave, Chou-navet \& Chou-rave, Navet \& Rave, Radis d'hiver, Pomme de terre, Betterave fourragère, Carotte fourragère, Maïs, Ail, Oignon. Concours ouvert à tous les jardiniers. Pas plus de neuf légumes par jardinier. Un spécimen par légume. À présenter avec feuilles, tiges et racines si possible. Ils seront jugés sur poids et aspects) ou consulter la météo dans les journaux locaux (Sur fond de ciel chaotique, pluies et averses se déclencheront, parfois accompagnées d'un coup de tonnerre dans l'après-midi) ${ }^{53}$.

Nous sont ainsi proposées autant de scènes de la vie de province, centrées sur quelques échantillons dérisoires d'une humanité fin de $\left(\mathrm{XX}^{\mathrm{e}}\right)$ siècle, apparemment elle-même parvenue en fin de parcours ou de partie, et enlisée dans une morne oisiveté décollée de tout grand dessein, qu'accusent encore les touches d'incongruité distillées par le romancier. Le dimanche paraît déteindre sur les autres jours de la semaine - est-ce là le dimanche de la vie? - et l'ennui de Baumgartner semble non seulement contagieux mais emblématique d'une forme de malaise dans la civilisation. Flaubert, de nouveau, n'est pas loin : que l'on songe à ce «ton gris, cette couleur de moisissure d'existence de cloportes ${ }^{54}$ » évoquée à propos de Madame Bovary - sans oublier, sur fond de commun paradigme agraire, un possible rapprochement entre l'illustre scène des comices agricoles et le concours de gros légumes...

Certes, et en dépit de telle mention critique relative aux conséquences des accords de Schengen (p. 181), il serait très excessif de lire dans Je m'en vais une virulente condamnation de la société contemporaine. Mais, à

52. Bruno Blanckeman, Les Récits indécidables, op. cit., p. 72 et suiv.

53. Jean Echenoz, Je m'en vais, op. cit., p. 172-173.

54. D'après la notation des frères Goncourt dans leur Journal, en date du 17 mars 1861. 
l'inverse, l'expression de ce toedium vitae collectif, quand bien même vidée de tout pathos par l'ironie, ne doit pas non plus être occultée, d'autant qu'elle permet de spécifier la tonalité des romans echenoziens : loufoque, soit, mais certainement pas joyeuse : bien plutôt douce-amère.

Enfin, reste à préciser que ce travail de modalisation ethnographique, somme toute discret en dépit de la fréquence des notations ponctuelles qui l'actualisent, se prolonge en une activité de médiation ontologique. Ici comme ailleurs, ce qui vaut pour le tout (le corps social), vaut aussi pour la partie (chacun des membres qui le composent). À la torpeur morbide qui pèse sur une " communauté » privée de tout projet collectif d'ampleur, répond à l'échelle individuelle la prégnance des sensations de manque, de perte, de chute ${ }^{55}$; ce qui se traduit tout particulièrement dans le parcours narratif du protagoniste, Ferrer, placé sous le signe de l'omniprésence de l'absence, si l'on ose dire. Son éducation sentimentale est un cinglant échec, sur fond de stagnation voire d'enfermement, ce que signale la structure circulaire du roman, elle-même exhibée par la reprise littérale de la phrase d'ouverture en guise de dernier(s) $\operatorname{mot}(\mathrm{s}):$ : je m'en vais ». D'où la tentation de voir en Ferrer un personnage emblématique de l'homme contemporain, qui face au monde et à ses habitant(e)s a depuis longtemps perdu son latin. «Fé/elix »? L'étymologie est trompeuse, et doit se lire ici sur le mode de l'antiphrase. En définitive, l'ironie de Jean Echenoz se révèle sinon plus grinçante, du moins plus douloureuse qu'il n'y paraît, qui use de tours et de détours lexicaux, syntaxiques et narratifs pour dire sans pathos l'insoutenable légèreté de l'être au présent.

Au terme de ce parcours, l'œuvre romanesque de Jean Echenoz, dont Je m'en vais me semble constituer un exemple caractéristique, frappe par la solidarité de ses diverses strates constitutives, autant dire par sa cohésion, qu'il est tentant de rapporter à un projet sous-jacent. Y incite notamment la forte lucidité critique de l'écrivain, évidente tant dans ses déclarations que dans la facture même de ses romans, dont l'esthétique stratifiée, gage d'une connaissance aiguë de l'histoire du genre et de ses formes, superpose harmonieusement les niveaux de sens, donc au moins potentiellement les niveaux de lecture. Le roman selon Jean Echenoz se situe ainsi aux antipodes de quelque naïveté que ce soit, et échappe par là même à toute dérive régressive, en vertu d'une pratique avisée de la narration, qui de buissonnante ne craint pas de se faire buissonnière, empruntant maints chemins de traverse qui n'en ramènent pas moins toujours à une intrigue orchestrée avec virtuosité. En résulte pour le lecteur une expérience esthétique des plus stimulantes où, après plusieurs décennies de douloureux célibat, peuvent enfin être consommées les noces de la distanciation réflexive et de la participation projective, c'est-à-dire aussi des jouissances

55. Bruno Blanckeman, «En théorie, “je m'en vais” », op. cit., p. 82-83. 
stratégique et esthétique. Réhabilitant ainsi, à plus d'un titre et en plus d'un sens, dans le cadre d'une pratique d'écriture résolument fictionnelle, et assez largement décomplexée, la notion de transmission narrative ${ }^{56}$, le roman echenozien apparaît emblématique de cette renarrativisation plus généralement à l'œuvre dans la littérature contemporaine, qui du retour en arrière n'a que les apparences, trompeuses. Cette salutaire rupture avec toute vision téléologique de l'Histoire, littéraire ou non, tient bien plutôt, en particulier chez Jean Echenoz, à un art consommé du pas de côté.

Frank Wagner

Université de Haute Bretagne - Rennes 2

56. Sur ce point, qu'on me permette de renvoyer à Frank Wagner, «Retours, tours et détours du récit (aspects de la transmission narrative dans quelques romans français contemporains) », Poétique, no 165, février 2011, p. 3-20. 